\section{ORIGINAL RESEARCH}

\author{
A.H. Aiken \\ P. Mukherjee \\ A.J. Green
}

C.M. Glastonbury

\title{
MR Imaging of Optic Neuropathy with Extended Echo-Train Acquisition Fluid-Attenuated Inversion Recovery
}

BACKGROUND AND PURPOSE: XETA, also known as Cube, is a relatively new 3D FSE sequence that can be used to perform whole-brain FLAIR T2-weighted imaging at isotropic high spatial resolution. This high-resolution volumetric imaging, coupled with both fat and fluid suppression, permits ideal evaluation of optic nerve anatomy and signal intensity; therefore, we hypothesized that XETA FLAIR would be useful for the detection of T2 signal-intensity abnormality in the optic nerve. Our purpose was to evaluate the sensitivity of XETA FLAIR for this abnormality and to compare it with the coronal FSE T2 FS.

\begin{abstract}
MATERIALS AND METHODS: After obtaining approval of the institutional review board, 2 CAO neuroradiologists retrospectively reviewed all patients with a clinical diagnosis of optic neuropathy who had undergone XETA FLAIR and standard orbital imaging from September 2006 to February 2009. Fifteen patients met these criteria and underwent the following sequences: XETA FLAIR, coronal FSE T2 FS, and T1-weighted postgadolinium sequences with FS.
\end{abstract}

RESULTS: Signal-intensity abnormality was identified on the correct side of the patient's vision loss in all 15 patients on XETA but in only 11/15 patients on the coronal FSE T2-weighted imaging. Reviewer 1 perceived the signal-intensity abnormality better on the XETA versus T2-weighted imaging in 10/15 patients, and reviewer 2 , in 9/15 patients. Neither reviewer visualized any of the imaging better by using the conventional coronal FSE T2 FS sequence.

CONCLUSIONS: XETA FLAIR was more sensitive than coronal FSE T2 FS for identifying abnormal signal intensity within the optic nerves in patients with optic neuropathy.

\begin{abstract}
ABBREVIATIONS: $\mathrm{CAQ}=$ Certificate of Added Qualification; Cor $=$ coronal; $E T L=$ echo train length; FLAIR = fluid-attenuated inversion recovery; FS = fat suppression; FSE = fast spin-echo; MS = multiple sclerosis; $P G=$ postgadolinium; $R 1=$ reviewer $1 ; R 2=$ reviewer $2 ; \mathrm{STIR}=$ short tau inversion recovery; SPIR = selective partial inversion recovery; T1W $=$ T1-weighted imaging; $\mathrm{T} 2 \mathrm{~W}=\mathrm{T} 2$-weighted imaging; $\mathrm{VEP}=$ visual-evoked potentials; XETA $=$ extended echo-train acquisition
\end{abstract}

0 ptic neuritis is one of the most common causes of injury to the optic nerve in young people and is due to inflammatory demyelinating injury to the retrobulbar nerve. Clinically, patients with optic neuritis have painful subacute visual loss associated with variable but often dramatic degrees of recovery. Noninfectious causes for optic neuritis are associated with a risk of developing disseminated demyelinating disease (ie, MS and neuromyelitis optica). Optic neuropathy is a more general term that includes both inflammatory and noninflammatory etiologies. Standard evaluation of a patient with suspected optic nerve injury includes clinical history and an ophthalmologic examination, which are usually sufficient for the diagnosis of uncomplicated acute optic neuritis. In demyelinating disease, VEPs can document chronic or subclinical injury to the optic nerve. However, VEPs are not always available, and clinical findings may be confounded by the presence

\section{Received June 1, 2010; accepted July 27.}

From the Departments of Neuroradiology (A.H.A., P.M., C.M.G.) and Neurology (A.J.G.), University of California, San Francisco, California; and Department of Neuroradiology (A.H.A.), Emory University Hospital, Atlanta, Georgia.

Paper previously presented at: Annual Meeting of the American Society of Neuroradiology, May 16-21, 2009; Vancouver, British Columbia, Canada.

Please address correspondence to Ashley H. Aiken, MD, Radiology Department, Emory University Hospital, 1364 Clifton Rd, Suite BG 26, Atlanta, GA 30322; e-mail: ashley.aiken@emoryhealthcare.org

DOI 10.3174/ajnr.A2391 of cataracts, outer retinal injury, postgeniculate injury, and technical error.

MR imaging is useful in cases of optic nerve injury with atypical clinical features or inconclusive VEP measurements and also may help to predict those patients who will ultimately develop MS. ${ }^{1} \mathrm{MR}$ imaging also allows exclusion of other lesions such as tumor or vasculitis that may mimic optic neuritis. $^{2}$ MR imaging analysis of the length and position of the affected segment may also provide prognostic information. ${ }^{3}$ Furthermore, correct identification of T2 abnormality within the optic nerve on MR imaging serves as a reliable marker for injury to the optic nerve. Improved methods for imaging the optic nerve may make MR imaging a more important element in the evaluation of optic nerve disorders, especially in chronic injury.

One of the challenges of optic nerve imaging is the surrounding orbital fat, due to its high signal intensity and chemical shift artifacts. Therefore, several studies have shown benefits from FS sequences. ${ }^{4-6}$ The use of FS with a STIR, frequency-specific SPIR, or T2-weighted FSE sequence with FS improves diagnostic sensitivity. Heavy T2-weighting can also improve accuracy, but as T2-weighting increases, the CSF signal intensity also increases, which can produce partial volume averaging artifacts, obscuring signal-intensity abnormality within the optic nerve. This problem is of particular impor- 
tance when imaging the optic nerve, where the high signal intensity of perineural CSF and the small cross-sectional area of the nerve cause difficulty in MR imaging interpretation. ${ }^{7} \mathrm{~A}$ solution to these problems would be to combine fat and fluid suppression for imaging the optic nerve. Jackson et $\mathrm{al}^{8}$ previously reported a hybrid SPIR-FLAIR sequence with both fat and fluid suppression that allowed considerable advantages in the assessment of optic neuritis. We now report imaging the optic nerve with a new sequence, XETA FLAIR, which also combines fat and fluid suppression.

The XETA, also known as Cube on GE Healthcare scanners (Milwaukee, Wisconsin), is a relatively new 3D FSE sequence that can be used to perform whole-brain FLAIR T2-weighted imaging at isotropic high spatial resolution. ${ }^{9}$ Similar sequences available on scanners from other manufacturers include Sampling Perfection with Application Optimized Contrasts by Using Different Flip Angle Evolution on Siemens systems (Erlangen, Germany) and VISTA on Philips Healthcare systems (Best, the Netherlands). Our standard protocol uses the optional chemically-selective FS, in which an adiabatic spectral inversion pulse is used to null fat. Any plane can be reconstructed from a single acquisition at high resolution, thus enabling reduction in total imaging time if multiple planes of FLAIR imaging are required. This high-resolution volumetric imaging coupled with both fat and fluid suppression permits evaluation of cranial nerve anatomy and signalintensity abnormality. At our institution, we have traditionally used 2 planes (axial and coronal) of FLAIR sequences ( 5 minutes each), which have now been replaced with XETA FLAIR (8 minutes), performed in the sagittal plane with coronal and axial reformats. Our purpose was to evaluate the sensitivity of XETA FLAIR for abnormal signal intensity in the optic nerve and to compare it with the coronal FSE T2-weighted sequence with FS.

\section{Materials and Methods}

After obtaining institutional review board approval, we retrospectively reviewed all patients with a clinical diagnosis of optic neuritis or optic neuropathy who underwent XETA FLAIR imaging from September 2006 to February 2009. Initially, we found 21 patients with both a clinical diagnosis of optic neuritis or optic neuropathy and XETA FLAIR imaging. However, 6/21 patients were excluded because they did not have standard orbital sequences for comparison. The remaining 15 patients were included in our study. All patients underwent a full ophthalmologic examination at presentation, including assessment of visual acuity and ophthalmoscopy. MR imaging was performed within 4 weeks of clinical presentation in 9 patients and after 4 weeks in the remaining 6 . The patient group consisted of 9 females and 6 males, ages ranging from 9 to 61 years. There was a clinical diagnosis of bilateral optic neuritis in 2 patients.

MR imaging was performed on a $1.5 \mathrm{~T}$ commercially available unit (Achieva 2.5, Philips Healthcare; HD 12.0 and HDx 14.0, GE Healthcare) in 12 patients and a 3 T commercially available unit (HDx 14.0, GE Healthcare) in 3 patients. All patients had the following sequences: XETA FLAIR, coronal T2 FS, and axial and coronal T1-weighted postgadolinium with FS. XETA parameters were as follows: TR, $6000 \mathrm{~ms}$; TE, minimum; ETL, 140 ms; NEX, 1 with 1.0-mm section thickness; 25 -cm FOV; $56 \times 256$ matrix interpolated to $512 \times 512$; and $2 \mathrm{D}$ autocalibrating reconstruction for Cartesian parallel imaging. An adiabatic spectral inversion pulse was used to null fat. The FOV covered the orbits and whole brain. Coronal FSE T2 FS sequence parameters were as follows: TR, $3000 \mathrm{~ms}$; TE, $102 \mathrm{~ms}$; ETL, 8 with FS; section thickness, $4 \mathrm{~mm}$ (skip, $1 \mathrm{~mm}$ ); and $256 \times 256$ matrix. T1-weighted postgadolinium parameters were the following: TR, $600 \mathrm{~ms}$; TE, minimum; NEX, 2 with FS; section thickness, $3 \mathrm{~mm}$ (skip, $0 \mathrm{~mm}$ ); and $256 \times 192$ matrix.

\section{Image Review}

Two CAQ-certified neuroradiologists ( 4 and 10 years' experience respectively) were blinded to the clinical history and side of vision loss. Each reviewed the studies for the presence and degree of signal-intensity abnormality on the XETA FLAIR versus the FSE T2 FS sequences. They used a subjective simple grading system: grade 0 , no signalintensity abnormality; grade 1 , subtle signal-intensity abnormality; and grade 2, gross signal-intensity abnormality. "Abnormality" was defined as increased signal intensity within the optic nerve compared with that of white matter or a combination of increased signal intensity and swelling. Postgadolinium T1-weighted sequences were also analyzed for the presence of enhancement. The length of abnormal the signal intensity was measured on the XETA axial reformatted images, and the location of signal-intensity abnormality (intraorbital, intracanalicular, or intracranial) was recorded.

\section{Results}

All 15 patients had optic neuropathy. Of these, 13 patients had a clinical diagnosis of optic neuritis, and 2 patients had a noninflammatory optic neuropathy. There was a clinical suspicion for or diagnosis of demyelinating disease in 12/13 patients with inflammatory optic neuritis, including a known diagnosis of MS in 4/12 and clinically isolated syndrome in $8 / 12$. The other patient with optic neuritis had an infectious optic neuritis secondary to histoplasmosis. Optic neuropathy was diagnosed in the remaining 2 patients, with hyperostosis causing compressive neuropathy in 1 patient and noninflammatory optic neuropathy in 1 patient.

\section{Subjective MR Imaging Appearances}

Abnormal signal intensity was demonstrated in all clinically affected optic nerves and was not seen in any asymptomatic nerve on the XETA FLAIR sequence as judged by both reviewers. The absence of perceived abnormal signal intensity in any normal nerve served as an internal control. There was a clinical diagnosis of bilateral optic neuropathy in 2 patients and unilateral optic neuropathy in the remaining 13 patients. In the 2 patients with bilateral abnormality, each had an acute optic neuritis as evidenced on imaging by $\mathrm{T} 2$ hyperintensity, swelling, and enhancement. Each also had contralateral chronic neuritis as evidenced on imaging by atrophy and signal-intensity abnormality, without enhancement. In all 9 patients imaged within 4 weeks of onset of symptoms, the nerve appeared normal-sized or enlarged and demonstrated enhancement postgadolinium, but the 6 patients who presented beyond 4 weeks did not have enhancement on postgadolinium sequences. Signal-intensity abnormality was correctly identified on the side of the patient's vision loss in all 15 patients on XETA FLAIR images, but in only $11 / 15$ patients on the T2weighted sequences (Fig 1). In addition, the degree of signalintensity abnormality differed significantly between the $2 \mathrm{~T} 2$ weighted techniques. XETA showed grade 2 or obvious signalintensity abnormality in most patients (11/15), whereas the 


\section{Presence of Signal Abnormality}

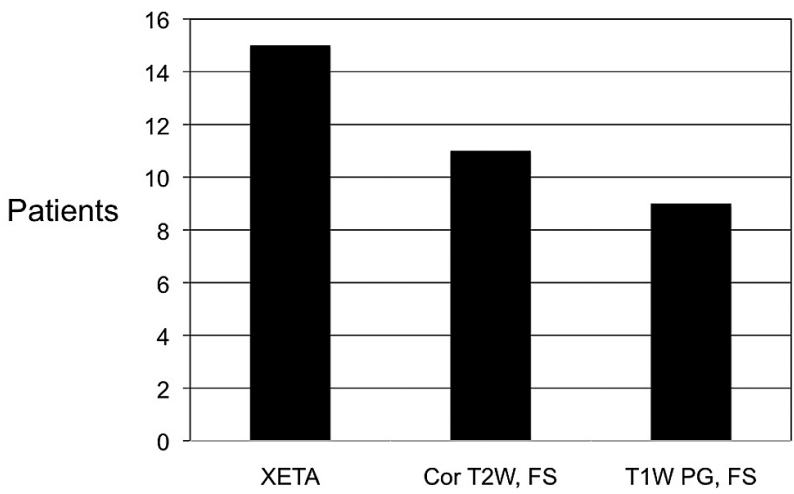

Fig 1. Graph shows signal-intensity abnormality in all patients on XETA on the correct side of the vision loss, but in only 11/15 on T2-weighted imaging. All 9 patients who presented acutely showed enhancement, but the 6 patients who underwent MR imaging after 4 weeks did not have enhancement. Two patients with negative T2 presented in the chronic period. These patients had no enhancement, so they would have been missed on all of our standard orbital sequences without XETA.

\section{Degree of Signal Abnormality}

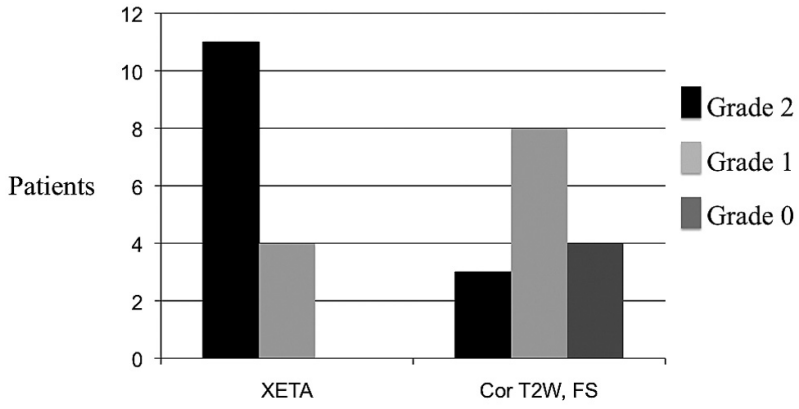

Fig 2. This graph shows the difference in the degree of signal-intensity abnormality. XETA shows grade 2 (obvious T2 hyperintensity) in most patients, whereas the coronal T2 FSE imaging shows only grade 1 (subtle T2 hyperintensity) in most patients and grade 0 (no signal intensity abnormality) in 4 patients.

standard coronal T2 imaging showed only grade 1 or subtle hyperintensity in most patients $(8 / 15)$ and grade 2 or obvious hyperintensity in only a small minority (3/15) (Fig 2).

The results of the individual interpretations are summarized in the Table. Reviewer 1 scored 11/15 XETA images as grade 2 (obvious abnormality), 4/15 as grade 1 (subtle abnormality), and $0 / 15$ as grade 0 (no abnormality seen). Reviewer 1 scored only 3/15 T2-weighted images as grade 2, 7/15 as grade 1 , and $5 / 15$ as grade 0 . Reviewer 2 scored 10/15 XETA images as grade 2 , five/ 15 as grade 1 , and $0 / 15$ as grade 0 . Reviewer 2 scored only 4/15 T2-weighted images as grade 2, eight/15 as grade 1 , and $3 / 15$ as grade 0 . Reviewer 1 perceived the signalintensity abnormality better on the XETA versus T2-weighted imaging in 10/15 cases and reviewer 2, in 9/15 cases (Figs 3 and 4). None of the cases were better visualized by either reviewer with the coronal FSE T2 FS sequence. There were no falsepositives on the XETA FLAIR sequence or the coronal FSE T2 sequence.

Two cases of isolated intracranial segment disease were seen only on the XETA images (Fig 5). The average length of signal-intensity abnormality was $2.15 \mathrm{~cm}$, ranging from 0.5 to $3.6 \mathrm{~cm}$. Twelve of 15 patients had a large intraorbital component, and $7 / 15$ had a combined intraorbital/intracanalicular
Results for individual interpretations for both reviewers

\begin{tabular}{|c|c|c|c|c|c|c|}
\hline \multirow[b]{2}{*}{ Sequence } & \multicolumn{2}{|c|}{ Grade 0} & \multicolumn{2}{|c|}{ Grade 1} & \multicolumn{2}{|c|}{ Grade 2} \\
\hline & $\mathrm{R} 1$ & $\mathrm{R} 2$ & $\mathrm{R} 1$ & $\mathrm{R} 2$ & $\mathrm{R} 1$ & R2 \\
\hline XETA & 0 & 0 & 4 & 5 & 11 & 10 \\
\hline T2 FS & 5 & 3 & 7 & 8 & 3 & \\
\hline
\end{tabular}

component. Only $1 / 15$ had isolated intracanalicular disease, and only $2 / 15$ patients had isolated intracranial nerve involvement. Both cases of isolated intracranial segment disease were seen only on XETA images.

\section{Discussion}

The benefits of FS techniques are well-known for orbital imaging and especially for imaging the optic nerve. ${ }^{2,5,6}$ Despite their widespread use, FS techniques, such as STIR, SPIR, and T2 FSE FS, all have the production of high signal intensity from CSF in the perineural sheath. High signal intensity from CSF poses 2 major problems: First, optic nerve signal-intensity abnormalities are made less conspicuous by volume averaging with CSF. Second, the surrounding CSF creates limitations on T2-weighting because the signal intensity from the fluid rises at a faster rate than that of the optic nerve. Furthermore, optic nerve sheath dilation has been shown in the setting of acute optic neuritis, compounding the problem of volume averaging from adjacent CSF signal intensity. ${ }^{7}$ Jackson et $\mathrm{al}^{8}$ showed the added advantages of combining fluid and fat suppression in optic nerve imaging with a combined SPIR-FLAIR technique. The authors stressed the benefits of the SPIR-FLAIR sequence resulting from an increased TR and the consequent increased ratio of signal intensity between neuritic and nonneuritic nerve segments and clearer delineation of the optic nerve due to the absence of signal intensity from perineural CSF. However, the SPIR-FLAIR sequence is prone to artifacts from field inhomogeneity, flow, and blurring due to the use of long echo trains. ${ }^{8}$

XETA or Cube FLAIR is a new 3D FSE sequence that can be used to perform whole-brain FLAIR T2-weighted imaging at isotropic high spatial resolution. XETA is a technique that combines the FSE technique, with its desirable T2-weighted contrast and insensitivity to off-resonance artifacts, and 3D volumetric acquisition, with its inherent high signal intensityto-noise ratio and the potential for very thin sections and ultimately isotropic resolution. XETA was developed with short echo spacing, optimized for very long echo-train readout, enabling fewer TRs to collect all the data. Second, it uses a technique for self-calibrated 2D-accelerated parallel imaging, thereby using fewer echoes to encode more resolution. Finally, by using a novel technique of refocusing flip angle modulation, XETA constrains relaxation during the long readout and voids the blurring that is commonly associated with long echo trains as noted in the SPIR-FLAIR imaging.

The optional chemically-selective FS uses an adiabatic spectral inversion pulse to null fat. This high-resolution volumetric imaging coupled with both fat and fluid suppression permits evaluation of cranial nerve anatomy and signal-intensity abnormality and is ideal for evaluation of optic nerve signal intensity. Very long echo trains and 2D-accelerated parallel imaging combine to vastly improve efficiency, making isotropic acquisition clinically realistic with XETA FLAIR. The 

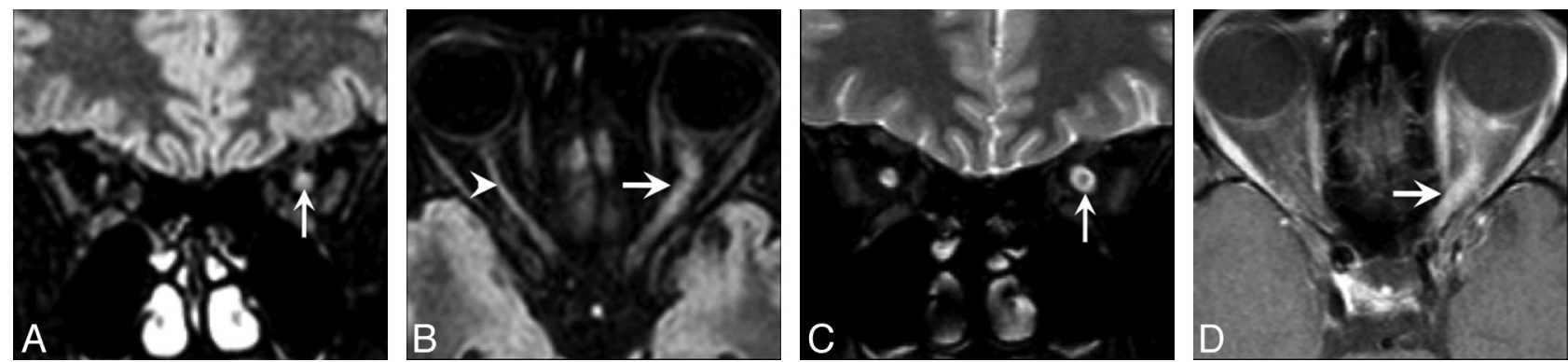

Fig 3. A 30-year-old woman presented with acute left optic neuritis. She had a history of contralateral (right) optic neuritis with persistent decreased visual acuity and an ultimate diagnosis of chronic relapsing inflammatory optic neuropathy. $A$ and $B$, Coronal $(A)$ and axial $(B)$ XETA reformations show obvious swelling and T2 hyperintensity in the left optic nerve (arrow). Of note, this is 1 of the 2 cases with bilateral abnormality. The right optic nerve is atrophic and also hyperintense secondary to chronic optic neuritis. $C$, The surrounding perineural CSF makes it difficult to perceive the T2 signal-intensity abnormality within the left optic nerve on this standard coronal T2 FSE FS image (arrow). D, Axial T1-weighted postgadolinium image shows enhancement of the left optic nerve (arrow).

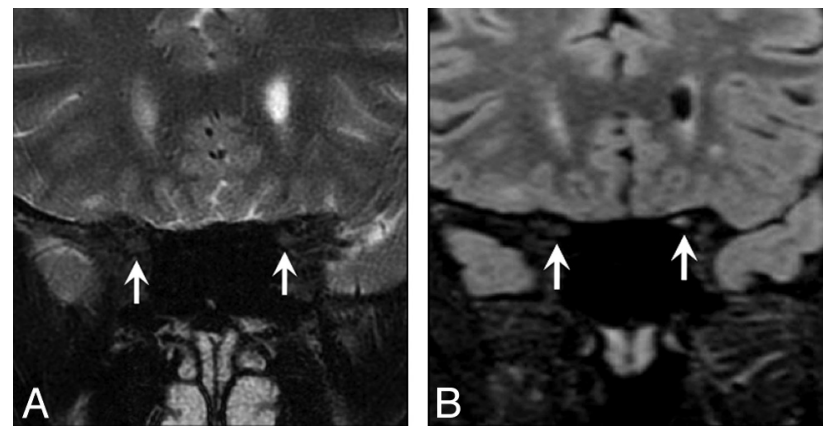

Fig 4. A 39-year-old man with subacute left optic neuritis but persistent decreased visual acuity. This was a clinically isolated syndrome, which carries a risk for the subsequent diagnosis of MS. A, Mildly motion-degraded coronal T2 FSE images do not show any signal-intensity abnormality within the optic nerves (arrows). B, Coronal XETA image easily shows hyperintensity within the intracanalicular portion of the left optic nerve as compared to the right optic nerve (arrows).
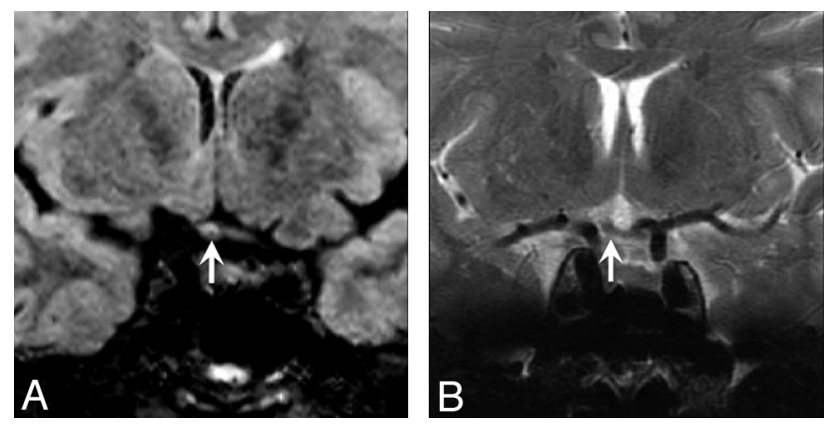

Fig 5. A 51-year-old man with a history of recurrent unilateral optic neuritis with chorioretinal atrophy on the right. $A$, Coronal XETA reformation nicely shows T2 hyperintensity in the right optic chiasm (arrow) in this unusual case of isolated intracranial segment disease. B, Coronal T2 FSE does not show the signal-intensity abnormality (arrow), likely because the surrounding CSF T2 hyperintensity obscures it.

variable flip refocusing pseudo-steady state sequence controls signal-intensity decay to produce sharp clear images reformatted in any plane.

We found that XETA is also particularly helpful to perceive signal-intensity abnormality in noninflammatory optic neuropathy and subacute-chronic optic neuropathy, where there may be no gadolinium enhancement. Youl et $\mathrm{al}^{3}$ studied the pathophysiology of acute optic neuritis and the timing of gadolinium leakage. Enhancement, signaling leakage of gadolinium across the blood-brain barrier, was a consistent finding in the acute lesion, but gadolinium leakage had ceased in 9/11 nerves imaged 4 weeks after the onset of acute symptoms. Our finding of no gadolinium enhancement in the 6 patients imaged after 4 weeks, are congruent with the study of Youl et al. ${ }^{3}$ The lack of gadolinium enhancement in subacute and chronic cases also emphasizes the importance of XETA, because 2/6 patients also had no signal-intensity abnormality and were false-negatives on our standard T2-weighted sequence. The signal-intensity abnormality was only perceived on XETA in these 2 cases.

Our study also suggests that XETA may also be particularly useful for intracranial segment disease. We had 2 patients with isolated intracranial segment disease, and neither patient had signal-intensity abnormality perceived on the standard coronal T2-weighted sequence. We hypothesize that the surrounding CSF signal intensity in this location decreases the conspicuity of T2 hyperintensity.

Another added advantage of XETA over our conventional coronal FSE T2 FS orbital sequence is that XETA includes whole-brain imaging that can be reconstructed in any plane. This is ideal for evaluating demyelinating plaques in MS and is arguably the most important component of imaging for optic neuritis. In the Optic Nerve Treatment Trial, the 5-year risk of developing MS was $16 \%$ in patients with normal brain MR imaging findings, $37 \%$ with $1-2$ lesions, and $51 \%$ with $\geq 3$ lesions. At 10 years, the only statistically significant difference was between no lesions (22\% risk) and $\geq 1$ lesions (56\% risk). ${ }^{10}$ Morrissey et al ${ }^{11}$ also showed that the risk of developing MS was much higher in patients with abnormal initial MR images (approaching 100\% at long-term follow-up), and the risk of developing MS remained low in patients with normal MR imaging findings. Therefore, MR imaging during an acute episode of optic neuritis has the unique ability to help predict the likelihood of developing MS after optic neuritis. The use of XETA FLAIR may reduce imaging time because it could be used for evaluation of the optic nerve and whole-brain imaging. Any plane can be reconstructed from a single acquisition at high resolution, thus enabling further reduction in total imaging time if multiple planes of FLAIR imaging are required. XETA FLAIR imaging may also be useful for documenting chronic optic injury and could reduce the need for evaluation by a clinical specialist.

The primary limitations of our study include the small sample size and the lack of comparison with healthy controls. However, reviewers were blinded to the clinically abnormal side. Therefore, in the 13 patients with unilateral abnormality, 
a clinically normal contralateral optic nerve served as an internal control. Our study strongly suggests that XETA is superior to standard coronal FSE T2 FS for imaging the optic nerve. However, a larger study with healthy controls would be helpful to confirm these findings. Furthermore, evaluations compared with functional assessments such as VEPs could be very useful for assessing the utility of XETA imaging for identifying clinically silent lesions of the optic nerve. XETA FLAIR is a very useful sequence in a combined MR imaging brain and orbits protocol because it not only provides multiplanar imaging for detection of intracranial demyelinating lesions but also has greater sensitivity for optic nerve signal-intensity abnormality than the conventional T2-weighted orbital sequence. Therefore, this single sequence could potentially replace 2 planes of whole-brain imaging and the orbital T2weighted sequence, streamlining imaging protocols.

\section{Conclusions}

The high-resolution volumetric imaging of XETA FLAIR enables excellent detection of abnormal optic nerve signal intensity and also allows multiplanar evaluation of the brain with 1 acquisition. The combined fat and fluid suppression is ideal for imaging the optic nerve, solving the problem of orbital fat and the surrounding T2 hyperintensity of CSF within the optic nerve sheath. XETA FLAIR has greater sensitivity for abnormal signal intensity than our standard coronal FSE T2 FS for imaging the optic nerves in patients with optic neuropathy.
Future studies with negative controls will be helpful to confirm these preliminary findings.

\section{References}

1. Brodsky MC, Beck RW. The changing role of MR imaging in the evaluation of acute optic neuritis. Radiology 1994;192:22-23

2. Gass A, Moseley IF, Barker GJ, et al. Lesion discrimination in optic neuritis using high-resolution fat-suppressed fast spin-echo MRI. Neuroradiology 1996;38:317-21

3. Youl BD, Turano G, Miller DH, et al. The pathophysiology of acute optic neuritis: an association of gadolinium leakage with clinical and electrophysiological deficits. Brain 1991;114(pt 6):2437-50

4. Johnson G, Miller DH, MacManus D, et al. STIR sequences in NMR imaging of the optic nerve. Neuroradiology 1987;29:238-45

5. Guy J, Mao J, Bidgood WD Jr, et al. Enhancement and demyelination of the intraorbital optic nerve: fat suppression magnetic resonance imaging. Ophthalmology 1992;99:713-19

6. Hendrix LE, Kneeland JB, Haughton VM, et al. MR imaging of optic nerve lesions: value of gadopentetate dimeglumine and fat-suppression technique. AJNR Am J Neuroradiol 1990;11:749-54

7. Hickman SJ, Miszkiel KA, Plant GT, et al. The optic nerve sheath on MRI in acute optic neuritis. Neuroradiology 2005;47:51-55

8. Jackson A, Sheppard S, Laitt RD, et al. Optic neuritis: MR imaging with combined fat- and water-suppression techniques. Radiology 1998;206:57-63

9. Busse RF, Hariharan $\mathrm{H}, \mathrm{Vu}$ A, et al. Fast spin echo sequences with very long echo trains: design of variable refocusing flip angle schedules and generation of clinical T2 contrast. Magn Reson Med 2006;55:1030-37

10. Beck RW, Cleary PA. Optic Neuritis Treatment Trial: one-year follow-up results. Arch Ophthalmol 1993;111:773-75

11. Morrissey SP, Miller DH, Kendall BE, et al. The significance of brain magnetic resonance imaging abnormalities at presentation with clinically isolated syndromes suggestive of multiple sclerosis: a 5-year follow-up study. Brain 1993; 116(pt 1):135-46 